\title{
Manchmal brauchen auch Politiker eine Zeit der Muße
}

\author{
Ein Gespräch mit Björn Engholm
}

Björn Engholm war von 1981 bis 1982 Bundesminister für Bildung und Wissenschaft und in den Jahren 1988 bis 1993 amtierender Ministerpräsident des Landes Schleswig-Holstein. Von 1991 bis 1993 war er Bundesvorsitzender der SPD. Im Interview mit Andreas Hoffelder (Zeitschrift für Politikberatung) spricht er über die aktuelle Lage der SPD, Großprojekte und Bürgerbeteiligung sowie die moderne Politikberatung.

\section{Herr Engholm, wie beurteilen Sie die aktuelle Lage der SPD?}

Sie ist mit $25 \%$ der Wählerstimmen ein bisschen bemitleidenswert, jedoch auf einem aufsteigenden Ast. Wenn ich auf die besseren Zeiten zurückblicke, dann gab es schon mal über $40 \%$, in Glücksfällen auch mal an die $50 \%$. Aber das hat die CDU in ihren guten Jahren durchaus auch erreicht. Nun sind SPD und CDU auf einen Wählersockel zurückgefallen, der es nicht immer leicht macht zu glauben, sie seien noch Volksparteien. Wenn man bedenkt, dass die Wahlbeteiligung heute bei Landtagswahlen und Kommunalwahlen inzwischen bei unter $50 \%$ liegt, teilweise noch nicht mal 40\%, dann erreicht die SPD mit 25\% gerade noch $10-13 \%$ der gesamten Wählerschaft. Die CDU hat dann, wenn sie etwas besser abschneidet als die SPD, 13\%-15\%. Das heißt, die beiden „großen “Volksparteien versammeln heute unter allen Wahlbürgern nicht mehr als ca. ein Viertel der möglichen Stimmen. Ich finde, das ist schon etwas Bejammernswertes.

\section{Wie kam es aus Ihrer Sicht dazu?}

Durch Veränderungen der Gesellschaft. Wir haben heute nicht mehr die großen Blöcke von Stammwählern. Die Sozialdemokraten hatten früher einen festen Block der gewiss 30\% ausgemacht hat. Arbeiterschaft, gewerkschaftlich organisiert, in sich solidarisch. Der ist in den vergangenen Jahren aber auseinandergefallen. Ein großer Teil der Arbeiterschaft besteht heute aus Angestellten und gewinnt mit dem Angestelltenstatus auch ein neues Bewusstsein.

Die Zahl der klassischen Arbeiter, der hart arbeitenden Menschen, ist viel kleiner geworden. Das ist ein Grund, warum sich die SPD nicht mehr auf ihren Standardblock verlassen kann, der immer zur Verfügung steht, wenn es ans Wählen geht. Der zweite Grund ist natürlich, dass die Sozialdemokraten in der jüngeren Vergangenheit Entscheidungen getroffen haben, die ihrer eigenen Klientel sehr weh getan haben. Die Schrödersche Politik mit Hartz IV, die ich jetzt im Einzelnen gar nicht auseinander nehmen will, hat das eigene Klientel hart getroffen. Viele von denen sind dann zu den Linken abgewandert, wobei auch viele hochfrustriert zu Nichtwählern geworden sind. Die kommen auch alle nicht mehr beliebig zurück.

\section{Der Niedergang der Industrieproduktion und Hartz IV sind also die Stolpersteine der SPD?!}

Ein dritter Punkt wäre noch, dass die Sozialdemokraten in den „Aufbruchszeiten“ in der Ära Willy Brandts oder auch zu Schmidts Zeiten ein sehr intensives Verhältnis zu den Künsten, der Wissenschaft und der Kultur hatten. Eine Klientel, welche zu großen Teilen links von der Mitte steht. Ungebundene, freie linke Liberale, wenn man so will. Dieses Verhältnis wurde von der Partei in der jüngeren Vergangenheit zu wenig gepflegt, wodurch uns ein großer Teil an geistigen und kulturellen Multiplikatoren verloren ging. Das war zu Brandts Zeiten noch anders. Auch hier in Schleswig-Holstein haben wir dieses Verhältnis in der regionalen Szene früher sehr intensiv gepflegt.

Das sind drei Punkte, von denen zwei objektiv schwer revidierbar sind. Die Zahl der Arbeiter wird sich nicht erhöhen und die nach links Abgewanderten werden nicht beliebig zurückkommen. Aber im Bereich Kultur und Wissenschaft wie auch in dem der jungen Entrepreneurs, die aus diesen Feldern kommen, könnte die SPD aktiver werden.

In der Politikwissenschaft spricht man davon, dass Parteien versuchen, die Mitte der Wähler anzusprechen? Gilt das immer noch?

Wenn man im Basisbereich nun nicht mehr die gewohnten Quoten herausholen kann, stellt sich automatisch die Frage „was ist die Mitte?“. Nun weiß aber natürlich keiner exakt, was eigentlich die Mitte ist. Für mich hieße das, sich an die jungen Aufsteiger wenden. Damit meine ich nicht diese drahtigen durchgestylten Jungs, die alle schon morgens um sieben vorm Laptop und um 22:00 immer noch im ICE vor demselben sitzen. Es gibt eine ganze Zahl von jungen Leuten, Entrepreneurs, die aus den Hochschulen kommen. In Lübeck gibt es davon viele. Die fangen mit einer oder mit zwei Personen an, neue Unternehmungen zu gründen. Die sind alles andere als konservativ, da sie geistig aufgeklärt sind. Um diese Szene muss die SPD sich kümmern, um die zukünftige Ökonomie, die zukünftige Dienstleistung und Wertschöpfung der Gesellschaft.

\section{Wurden linke Tendenzen in der SPD lange unterschätzt? Wenn man den Jusos zuhört, kann die Lafontaine-Affäre und die Abwanderung von SPD-Wählern zur Linkspartei nicht wirklich überraschen?}

Lafontaine/Schröder ist ein besonderes Psychodrama. Das hat nicht ausschließlich etwas mit politischen Positionen zu tun. Schröder gegen Lafontaine war ein Machtkampf, den Lafontaine verlor. Das hat er nie verwunden. Gerhard Schröder hat Lafontaine dann auch gehen lassen. Das ist mein Eindruck und 
dies war ein zentraler Fehler, den man beiden anlasten kann. Eine Partei muss eine mittlere Position haben, auch im Wirtschaftlichen, wie Schröder sie besitzt, und sie muss auch die linken Leute binden. Dafür war eben nun wieder Lafontaine mit Hilfe der Jusos der richtige Mann. Das ist mit seinem Weggang zerbrochen. Wenn ich an die großen Zeiten der Sozialdemokratie zurückdenke - Brandt, Schmidt, Wehner - die haben sich nicht immer geschätzt, aber sie haben um der Sache willen zusammengestanden. Aber diese Leute waren historische Größen, deren Motto hieß: Was auch immer uns persönlich trennt, darf der Partei oder der Sache nicht schaden. Diese Größe war zu Zeiten der Trennung zwischen Lafontaine und Schröder schwer erkennbar.

\section{Wer soll ihrer Meinung nach heute den Parteivorsitzenden wählen?}

Die in der SPD eingeschriebenen Mitglieder. Ich finde es nicht in Ordnung, wenn man hier beliebig die Pforten öffnet und die Wahl von Repräsentanten in den Parlamenten oder an der Parteispitze durch Außenstehende bestimmen lässt. Dafür haben wir Mitglieder, deren wichtigstes Recht darin besteht, über die Entscheidung für Repräsentanten die Linie der Partei substantiell mitzubestimmen. Wenn man ihnen das durch das Hinzunehmen von Auswärtigen streitig machen würde, verlören wir mehr Leute, da der politische „Mehrwert“ der Parteizugehörigkeit verloren ginge.

\section{Ist ein guter Parteivorsitzender auch immer ein guter Kandidat?}

Das ist nicht automatisch der Fall. Das kann in Glücksfällen mal sein, aber eine Partei zu führen, selbst wenn sie heute nur noch 500.000 Mitglieder hat wie die beiden Großen - es waren ja mal 1.000.000 - ist de facto ein Fulltime-Job. Ich war eine Zeitlang Ministerpräsident. Das ist ebenfalls ein Fulltime-Job. Nebenher dann noch ein Partei führen, geht eigentlich nicht. Wenn dann noch die Frage der Kanzlerkandidatur hinzukommt, überfordert es einfach.

Menschen in Spitzenpositionen in der Politik, Wirtschaft oder Kirche brauchen Zeiten der Muße. Die müssen Zeit finden, mal ein neues Buch lesen, Musik zu hören, sich mit Leuten außerhalb ihres Metiers austauschen. Bei der Ballung von verschiedenen Funktionen kommt das zu kurz. Die Leute werden am Ende mehr oder minder zu Getriebenen. Und ich möchte, dass sie treiben statt getrieben werden.

\section{Versuchen Sie noch Einfluss auf das Tagesgeschäft der Partei zu nehmen? Sind sie dort noch in irgendeiner Form beratend tätig?}

Nein, im parteilichen Sinne sitze ich noch im Beirat, der das SPD-Vermögen kontrolliert. Wir haben ja ein recht umfängliches Portfolio aus der Geschichte heraus. Die SPD hat durch Restitution das durch Kaiser, Hitler und die SED gestohlene Vermögen zurückerhalten. So haben wir heute einen Teil unse- rer früher durch Mitgliedsbeiträge erworbenen Besitztümer wieder. Das sind Zeitungsbeteiligungen, Druckereien, oder auch Reisebetriebe. Eine bunte Mischung - ein gut sortiertes Portfolio.

Ein anderes Thema: Wie nehmen Sie aus einiger Entfernung das Thema Stuttgart 21 wahr? Wie haben sie die Wahl in Baden-Württemberg und die Tatsache, dass die SPD dort als „kleiner Koalitionspartner“ agieren muss aufgenommen?

Wenn einer mitregiert, dann ist er „Mitsieger“, egal wie viel Prozent er bekommen hat. Aus der Distanz betrachtet finde ich es hoch aufregend, was dort in Stuttgart passiert. Es sind Ansätze zu neuen Bürgerbewegungen zu erkennen, also Bewegungen aus der Mitte heraus. Das hat es ja in der Geschichte lange nicht gegeben. Bei früheren Versuchen „Revolution zu machen “ und den liberalen Aufständen gab es so etwas. Da müssten die Parteien sehr sorgsam hinschauen. Zu dem Projekt Stuttgart 21 kann man eine ganze Philosophie entwickeln. Ich bin prinzipiell kein Anhänger von Großprojekten. Jene, die ich in meiner Geschichte miterlebt habe, verliefen alle nicht sehr glücklich.

\section{Welche waren das?}

Mammutuniversitäten, Großkonzertsäle, desaströse Wohnmaschinen, der Transrapid auf zu kleinen Strecken. Ich bin hier eher für die Kleinteiligkeit. Sie ist überschaubarer und intimer, sie schafft Bindungen, Identität. Am Ende wird der Bahnhof in Stuttgart 5 Mrd. und mehr kosten. Ob er diese Kosten wieder einspielt und ob es sich lohnt, dafür einen großen Teil der bürgerlichen Mitte in die Mangel zu nehmen, da bin ich skeptisch.

\section{Wie stehen Sie gerade in diesem Zusammenhang dem Thema Bürgerbeteiligung gegenüber?}

So viel Bürgerbeteiligung wie möglich! Aber nur soweit, wie das repräsentative System nicht in Frage gestellt wird. Ich bin ein Anhänger des repräsentativen Systems und je schwieriger und komplexer unsere Fragen heute im Zeitalter der Globalisierung werden, desto schwieriger wird es für Menschen über alles abzustimmen. Damit will ich gar nicht sagen, dass sie von einer Thematik weniger verstehen als ihre jeweiligen Repräsentanten in den Parlamenten. Aber ich brauche hinterher, wenn eine Entscheidung falsch war, immer einen Verantwortlichen. Bei einer falschen Bürgerentscheidung kann ich keinen Bürger dafür zur Rechenschaft ziehen. Trifft ein Parlament eine falsche Entscheidung, kann ich mit dafür Sorge tragen, dass die Hauptrepräsentanten nicht wieder gewählt werden. 
Sie haben gerade schon erwähnt, dass Sie generell kein Freund von Großprojekten sind. Wie beurteilen Sie Großproketen, die geographisch etwas näher liegen wie zum Beispiel die Elbvertiefung?

Bei der Elbvertiefung muss man anmerken, dass Hamburg zwar nicht nur vom Hafen lebt. Ich weiß nicht genau, wie hoch der Wertschöpfungsanteil des Hafens ist. Trotzdem ist es ein signifikanter Bevölkerungsanteil, der direkt oder mittelbar vom Hafen lebt - vom Beitrag des Hafens zum Weltimage Hamburgs ganz zu schweigen. Die Frage ist, was mit dem Hamburger Hafen passiert, wenn er sich von den Warenbewegungen in der Welt abkoppelt. Die Schiffe werden größer werden. Es werden bald keine 50.000 Tonner mehr laufen sondern 160.000 Tonner und größer. Wenn die Hamburger entscheiden, dass diese Schiffe nicht mehr in den Hafen kommen sollen, dann werden sie nach Antwerpen oder Rotterdam abwandern.

Also muss man abwägen, wie groß der Schaden ist und wie groß der Nutzen. Bei der Elbvertiefung ist mein Eindruck allerdings, ich bin ja auch Aufsichtsratsvorsitzender vom Lübecker Hafen, dass der Hamburger Hafen Tonnage verliert, wenn sich die Stadt dagegen entscheidet.

\section{Als Zeitschrift für Politikberatung interessiert uns neben dem „was" auch das „wie“ der Beratung und der Kommunikation. Sehen Sie in der politischen Berichterstattung einen Trend hin zur „Skandalisierung“?}

Heute wird die Intimsphäre von Menschen medial viel leichter durchbrochen. Vor 30-40 Jahren, zu meiner politischen Frühzeit, hat man auch Spitzenpolitikern noch ein Leben nach Feierabend zugebilligt. Dieser Schonraum ist im Laufe der Zeit immer weiter eingeschränkt worden. Dort findet eine „Entprivatisierung “ statt, die Schäden in den Köpfen der Betroffenen auslöst. Man sieht sich heute nur noch vor. Man geht nicht mehr in Damenbegleitung abends mal ein Glas Wein trinken, weil man genau weiß, am Tisch gegenüber sitzt der nächste Redakteur und morgen früh bin ich mit Großaufnahme im Boulevard.

\section{Das schließt an das Thema Muße an.}

Es beginnt eine Art Schere im Kopf, die die Politiker daran hindert, Muße und auch ein bisschen Genuss zu finden. Sich übermäßig vorzusehen, political correct zu sein, ist sehr anstrengend. Diese totale Political Correctness hat etwas Schreckliches. Sie macht aus Menschen geölte Maschinen. Das Überborden medialer Ausspähung der Privatsphäre von Menschen bzw. Politikern hat negative Folgen. Allerdings muss man sagen, dass es in Deutschland bei weitem noch nicht so schlimm ist wie in England. Da ist das deutsche Mediensystem im Großen und Ganzen zurückhaltender. Aber das man aus jeder Kleinigkeit eine Sensation macht, eine Enthüllung, eine Entdeckung oder einen Skandal, auch wenn es nicht direkt etwas mit Politik zu tun hat, ist bedauerlich.
Akribisch recherchierte Sachen im politischen oder wirtschaftlichen Kontext, das sind ja nicht sehr viele, finde ich völlig in Ordnung. Wenn sie die erwischen, die Schindluder treiben, dann müssen die dafür bluten. Hier ist es auch legitim, wenn dies durch Journalisten aufgedeckt wird. Leider gibt es davon zu wenige. In Deutschland sind es vielleicht noch ein Dutzend, da sich nur noch wenige Zeitungen solche Journalisten leisten.

\section{Haben diese direkten Angriffe Auswirkungen auf das politische Tagesgeschehen? Werden durch die daraus resultierende Vorsicht der politischen Akteure inhaltlich schwierige Fragen eher ausgeblendet, um sich nicht die Finger zu verbrennen und angreifbar zu machen?}

So richtig knorrige Typen gibt es heute ja kaum noch in der Politik. Ich war ja nun solider Norddeutscher, nicht besonders knorrig. Aber da gab es von Wehner bis Strauß einfach einen Typus, die sich nicht darum geschert haben, was irgendjemand von ihnen gedacht hat. Wenn ich bedenke, was da an Leben in der Bude war, wenn diskutiert wurde und die aufeinander trafen! Das gibt es heute nicht mehr. Heute kommen sie alle von denselben Hochschulen, haben dieselben Examina, jeder hat ein bisschen bei seiner Promotion geschummelt. Sie haben alle denselben Schneider und Haarschneider. Viele sind austauschbar geworden und das finde ich sehr schade.

Als ich Bildungsminister war, kamen sechs oder sieben Journalisten zur Pressekonferenz. Zwei Mikrophone, eine Kamera und drei Schreibende. Man konnte sich dann auch aussuchen, mit wem man was macht. Heute stehen bei jeder Kleinigkeit 20 Kameras da. Wenn man selbst darauf verzichtet, eine Antwort zu geben, und zwar aus dem einfachen Grund, weil man sie nicht kennt, dann beißt der Nachbar ins Mikrophon. Der Zwang, sich zu produzieren und dabei immer fröhlich, optimistisch, gut gekleidet und ausgeschlafen zu sein, ist groß geworden. Durch diesen Zwang, sich in jeder Minute omnipotent zu produzieren, geht eine markante Differenziertheit verloren.

\section{Welche Erfahrungen haben Sie in Ihrer Vergangenheit mit Krisenkommunikation gemacht? Worauf muss man achten?}

Das Einstellen auf eine Krise beginnt immer schon vor der Krise. Soziologen haben das eine vorbereitende Sozialisation genannt. Man muss im Hinterkopf haben, dass einem irgendwann mal etwas passieren kann. Und dann braucht man einen Wegweiser, wie man sich verhält. Jemand der sich gut auf die Jobsuche und das Vorstellungsgespräch einstellt, sich in die Leute hineinversetzt und gute Kenntnisse vom Unternehmen hat, der hat gute Chancen. Das ist vorbeireitende Sozialisation auf eine Situation, die kommt.

Das muss bei Politikern auch stattfinden. Es beginnt damit, dass man sich am Tag seiner Wahl hinter die Löffel schreibt, dass man nur für vier Jahre gewählt ist und man nach diesen vier Jahren abgewählt werden kann. Politik ist ein befristeter Job und Leute, die mit einer Anspruchshaltung daran gehen, 
dass sie mindestens vier oder fünf Legislaturperioden machen wollen, liegen einfach falsch. Die erwischt es beinhart, wenn sie beim zweiten Mal nicht wiedergewählt werden. Ein Stück Bescheidenheit als Verhaltenskonstante zu haben, ist von vornherein ratsam. Man muss wissen, dass man nicht auf Lebenszeit gewählt ist.

Darüber hinaus muss man sich bewusst machen, dass man auch nach einer Niederlage derselbe bleibt. Man benötigt ein sehr starkes Verhältnis zu sich selbst, eine ausgeprägte „IchIdentität“. Damit meine ich nicht, dass man immer durch die Gegend strahlen soll wie Herr zu Guttenberg. Sondern die Bescheidung zu wissen, wenn mir etwas passiert, muss ich das in Kauf nehmen, insbesondere wenn ich selbst daran schuld bin, ich bleibe ich selbst. Ein Politiker braucht dieses Grundverständnis von begrenztem Auftrag, unter ständiger Kontrolle des Volkes, mit der Möglichkeit zu scheitern. Wenn man das einmal begriffen hat, dann kommt man mit Krisen auch ganz gut zurecht.

Darüber hinaus benötigt man in Krisenzeiten Leute, die nicht aus dem engeren professionellen Umfeld stammen. Leute für die Diskussion über die Krisenbewältigung, die nicht selbst existenziell an einer bestimmten Antwort hängen. Ich hatte ja selber eine große Krise. Da waren für mich Menschen wichtig, die außerhalb des eigenen politischen Kontextes leben. Diese können den Blick von außen auf die Thematik werfen, weil sie einen extern schätzen. Wenn man sich nur mit dem kleinen „Küchenkabinett“ berät, mit dem man jahrelang zusammengearbeitet hat und in dem jeder weiß, wenn der "Alte“ geht, dann muss ich auch gehen, bekommt man keine vernünftige Krisenbewältigung hin. Hinzu kommt noch, wenn es wirklich um eine große existenzielle Krise geht, muss man auch professionelle Hilfe von außen in Anspruch nehmen. Das hat nichts Ehrenrühriges.

\section{Schaut man auf ihre Auseinandersetzung mit Barschel und dessen Methoden, stellt sich inzwischen die Frage, ob diese Gangart mittlerweile üblich geworden ist?}

Nein, ich denke in dieser Form hat sich das nicht wiederholt, jedenfalls nicht in Deutschland. Vielleicht war das auch eine Lehre aus dem damaligen Skandal. Man muss konstatieren, dass der politische Stil heute nicht mehr ganz so rau ist, wie er früher war. Wenn man bedenkt, was Heiner Geißler alles über Sozialdemokraten erfunden hat, mein Gott! Immerhin muss man sagen, dass er heute ja eine Art Ersatz- Jesus geworden ist. Da kann man ihm dann auch verzeihen. Aber diese Art der Konstruktion von Bösartigkeiten, bei denen man hofft, dass sie dem Gegner besonders wehtun und am Ende aus dem Gegner einen Feind machen, das hat eindeutig nachgelassen.

Da hat Schmidt sehr gut gewirkt in seiner Zeit. Er hatte zwar eine ganz spitze Zunge, aber letztendlich hatte er Achtung vor einem guten Gegner und hat ihn als Konkurrent und nicht als Feind betrachtet. Auch bei Kohl gab es Dinge, die nicht schön waren. Merkels Stil ist hingegen ein sehr verträglicher. Und Steinmeier und Steinbrück besitzen ebenfalls Stil, so dass ich insgesamt eine größere Contenance feststelle. Das macht es aber natürlich auch langweiliger. Aber dass Bösartigkeiten nicht mehr an der Tagesordnung sind, ist auf jeden Fall ein Gewinn.

\section{Ist die neu gefundene Contenance ein deutsches Politikphänomen oder kann man das auch im Ausland feststellen?}

Sieht man von einigen Tendenzen in der neueren Zeit ab, so hat es eigentlich gerade im skandinavischen Bereich immer ein relativ anständiges Miteinander gegeben. Es gibt inzwischen Rechtstendenzen im Norden, von denen ich nicht weiß, wo sie hinführen. Aber was insgesamt in Skandinavien in den letzten Jahrzehnten politisch passiert ist, war für mich immer Vorbildhaft. Sowohl den Umgang als auch die Lösungen betreffend.

England dagegen ist in diesem Sinne kein Vorbild. Seit Maggie Thatcher ist da etwas kaputt gegangen. Mit Reagan hat sich in Amerika leider auch vieles verändert. Das ist nicht mehr der alte „New-Deal“- und Fairplay-Gedanke, es ist straff kapitalistisch geworden in einer unschönen Form. Wenn man sich nur einmal anschaut, was die Tea-Party und die Republikaner in den USA veranstalten! In Zeiten, in denen der Staat bankrott ist, noch auf Steuersenkungen zu drängen, das ist hirnrissig. Dahingegen sind wir in Deutschland relativ gut davor. Die Holländer haben sich auch lange Zeit, bis auf die neuerlichen Rechtstendenzen, gut konstituiert. Auch Frankreich ist immer noch erkennbar eine politische Kulturnation. Unterm Strich sind aber gerade die Deutschen vorzeigbar. Es ist nicht die schlechteste Demokratie, die wir hier haben.

\section{Sie haben bei der Krisenkommunikation bereits angesprochen, dass man sich ab einem gewissen Punkt professionelle Hilfe holen, sich also politisch beraten lassen muss. Hat sich Politikberatung in der Vergangenheit verändert? Ab wann ist dieser Punkt erreicht, ab dem man sich professionelle Hilfe sucht, sei es nun inhaltliche oder strategische Hilfe?}

Es ist relativ schwer zu definieren, was Politikberatung im Einzelnen ist. Das war früher schon nicht leicht. Alles, was sich zu meiner aktiven Zeit Politikberatung nannte, war zu 75\% Lobbyarbeit. Ich vermute, dass es heute noch genauso ist. Das habe ich nie unter Politikberatung verstanden. Wir haben uns mal von Fachleuten außerhalb der Politik politisch beraten lassen.

Wir haben in Schleswig-Holstein eine Denkfabrik gegründet, als wir 1988 an die Regierung kamen. Die Prämisse war, dass wir uns als kleines Land im Norden in der Ecke von Deutschland, damals sogar ja noch mit innerdeutscher Grenze, neu definieren müssen. Mitglieder der Denkfabrik waren unter anderem der Chef von Bosch, der von Philips und der von Veba, die zwei Gewerkschaftsbosse der damaligen Zeit, ein Bischof, Wissenschaftler, die Chefs von Volvo und der Deutschen Bank usw. Wir haben alles eingeladen, unabhängig davon, wo die betreffenden Personen stehen und wie 
sie im Einzelnen denken. Über eineinhalb Jahre haben die Personen dann in verschiedenen Arbeitskreisen Dinge erdacht, an die wir niemals zu denken gewagt hätten. Das war auswärtige Beratung, wie ich sie mir vorstelle.

Wenn ich mir heute Unternehmensbosse ansehe, dann stelle ich fest, dass sie weit von der Politik entfernt sind. Viele sind auch weit vom Menschen entfernt. Ich habe manchmal den Eindruck, dass die keine Ahnung haben, wie Politik funktioniert. Deshalb gibt es ja auch so wenige Quereinsteiger in die Politik und die wenigen sind fast alle gescheitert. Ich kann mich noch an den ehemaligen Generalmanager von Krupp erinnern. Ein erstklassiger Mann, den Schmidt als One-DollarState-Secretary geholt hat. Er war es natürlich gewohnt, bei Krupp Entscheidungen zu treffen und dort hat es funktioniert. In der Politik musste er sich aber nun mit ganzen Stäben auseinandersetzen, mit Ministerien, Fraktionen, Arbeitskreisen. Er hatte nie die politischen Zusammenhänge gelernt. Von hierarchischer Entscheidungsverantwortung zu demokratischer Willensbildung: ein langer Weg, den nur sehr wenige schaffen.

\section{Wie stehen sie der „modernen Politikberatung“ gegenüber? Es gibt ja immer mehr Agenturen, die sich auf „Politikvermittlung“ spezialisiert haben und überhaupt nicht mehr den Anspruch haben, Wissen zu generieren.}

Das hängt alles damit zusammen, dass sich heute alles nach dem Gesichtspunkten der Nutzbarkeit, des Zweckes und der Effizienz richtet. Ich denke, die Kernaufgabe von Politikberatung muss sein, Zusammenhänge zu verdeutlichen. Eigentliche
Beratung ist daher ja ein mehr oder minder geistiger Prozess. Was heute stattfindet ist der Versuch, materielle Interessen möglichst schnell an den Mann oder an die Frau zu bringen. Argumente zu finden, wie man einen anderen aushebeln kann, der eben andere Interessen oder andere Ideen vertritt. Das, was sie eben geschildert haben, ist in diesem Sinne eigentlich keine Beratung mehr. Beratung war für mich immer ein machtfreier Prozess des sich Auseinandersetzens mit Dingen. Oder wie Kant uns so schön gelehrt hat, sich des Verstandes zu bedienen. Heute stehen alle immer unter immensen Zwängen. Sie müssen ihre Quartalsbilanzen vorlegen und wenn ein Unternehmen drei Prozent unter der Planung bleibt, rutscht der Kurs sofort weg. Also setzt man alles ein, um Ziele, Zwecke und Nutzen zu generieren. Das sind dann Durchsetzung von Kapitalinteressen und keine Beratung.

\section{Herr Engholm, vielen Dank!}

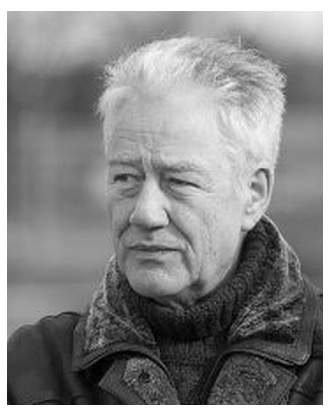

Björn Engholm ist 71 Jahre, war lange Jahre Vorsitzender des Kulturforums (der Sozialdemokratie) Schleswig-Holstein e. V. sowie Vorsitzender des Universitätsbeirates der Universität zu Lübeck und deren Ehrenbürger. Weiterhin ist er Mitglied der Treuhand-Kommission der SPD, des Kontrollgremiums über die Finanzen und Immobilien der Partei und Aufsichtsratsvorsitzender der Lübecker Hafengesellschaft.

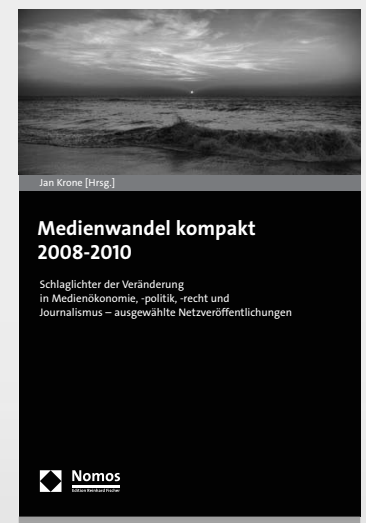

Medienwandel kompakt 2008-2010

Schlaglichter der Veränderung in Medienökonomie, -politik, -recht und Journalismus - ausgewählte Netzveröffentlichungen

Herausgegeben von Jan Krone

2011, 302 S., brosch., 29,-€, ISBN 978-3-8329-6263-0

Der Band greift den Medienwandel aus ökonomischer, politischer und journalistischer Perspektive der letzten zwei Jahre auf. Dazu werden ausgewählte Beiträge aus Netzveröffentlichungen herangezogen. Die Leser erhalten somit einen redaktionell gefilterten, kompakten Überblick über die Umbrüche der Medienlandschaft durch die Digitalisierung, das Internet. 